\title{
Effective Atomic Number Measurement with Energy-Resolved Computed Tomography Using Two-Dimensional “transXend” Detector
}

\author{
Hiraku Iramina ${ }^{1,2}$, Mitsuhiro Nakamura ${ }^{2,3}$, Takashi Mizowaki ${ }^{2}$, Ikuo Kanno ${ }^{{ }^{*}}$ \\ ${ }^{1}$ Department of Nuclear Engineering, Graduate School of Engineering, Kyoto University, Kyoto, Japan \\ ${ }^{2}$ Department of Radiation Oncology and Image-Applied Therapy, Graduate School of Medicine, Kyoto University, Kyoto, Japan \\ ${ }^{3}$ Division of Medical Physics, Department of Information Technology and Medical Engineering, Human Health Sciences, \\ Graduate School of Medicine, Kyoto University, Kyoto, Japan \\ Email: ^kanno@nucleng.kyoto-u.ac.jp
}

How to cite this paper: Iramina, $\mathrm{H}$., $\mathrm{Na}$ kamura, M., Mizowaki, T. and Kanno, I. (2018) Effective Atomic Number Measurement with Energy-Resolved Computed Tomography Using Two-Dimensional "transXend" Detector. International Journal of Medical Physics, Clinical Engineering and Radiation Oncology, 7, 61-73. https://doi.org/10.4236/ijmpcero.2018.71006

Received: December 29, 2017

Accepted: February 10, 2018

Published: February 13, 2018

Copyright (c) 2018 by authors and Scientific Research Publishing Inc. This work is licensed under the Creative Commons Attribution International License (CC BY 4.0).

http://creativecommons.org/licenses/by/4.0/

\begin{abstract}
Introduction: We have previously developed an effective atomic number $\left(Z_{\text {eff }}\right)$ measurement method using linear attenuation coefficients (LACs) obtained by energy-resolved computed tomography (CT) with one-dimensional (1D) detector. The energy-resolved CT was performed with a "transXend" detector, which measured X-rays as electric current and then gave X-ray energy distribution with unfolding analysis using pre-estimated response function (RF). The purpose of this study is to measure $Z_{\text {eff }}$ by the energy-resolved CT using a flat panel detector (FPD). Methods: To demonstrate a 2D transXend detector, we developed the stripe absorbers for the FPD. Eleven human tissue-equivalent material rods which were grouped into four material categories were measured by X-rays with $120 \mathrm{kVp}$ tube voltage, $2.3 \mathrm{~mA}$ tube current, and $1.0 \mathrm{~s}$ exposure time. $Z_{\text {eff }}$ is measured by the ratio of LACs with two different pseudo-monochromatic X-ray energies. RFs of each rod material were estimated by numerical calculation. First, we employed the RF estimated for the same rod material (self-RF scenario). Second, we employed the RF estimated for the different rod materials in the same material category (cross-RF scenario). The purpose of the cross-RF scenario was to find representative rod materials in each material category. Results: Upon the self-RF scenario, measured $Z_{\text {eff }} \mathrm{s}$ were systematically underestimated. Median relative error to theoretical $Z_{\text {eff }}$ was $-6.92 \%$ (range: $-7.89 \%--4.60 \%$ ). After normalizing measured $Z_{\text {eff }}$ s to the theoretical one for Breast, median relative error improved to $-0.75 \%$ (range: $-1.79 \%-+1.73 \%$ ). Upon the cross-RF scenario, the representative rod materials were found in two material categories. Conclusion: $Z_{\text {eff }}$ measurements were performed by energy-resolved CT using $2 \mathrm{D}$ transXend detector with numerically-estimated RF data. Normalized $Z_{\mathrm{eff}} \mathrm{s}$ for
\end{abstract}


all rod materials in the self-RF scenario were in good agreement with the theoretical ones.

\section{Keywords}

X-Ray, Computed Tomography, Energy Resolved, Unfolding, Effective Atomic Number

\section{Introduction}

In photon and particle radiation therapy treatment planning, single-energy computed tomography (CT) image is commonly used to distinguish materials inside a patient body and to calculate absorbed doses. A CT image is a distribution of linear attenuation coefficient (LAC) of each pixel. To calculate the dose in the patient body, LACs are converted to corresponding mass densities using a conversion table. However, estimated LACs for each pixel can be different from true LACs due to the beam-hardening effect: when polychromatic X-rays pass through a subject, the effective energy of X-rays increases because of the absorption of low energy X-rays. Also, estimated LACs of different materials would be similar and hard to distinguish in single-energy CT image. Thus, a mis-assignment of mass density may occur in single-energy CT measurement.

To make the dose calculation accurately, a Monte Carlo method has been implemented [1]. If the mis-assignment of mass density due to the beam-hardening effect happened, it can lead to significant dose errors: up to $10 \%$ error for $6-15$ megavoltage (MV) photons [2]. Recently, an effective atomic number $\left(Z_{\text {eff }}\right)$ attracts attention as an alternative value to LAC.

Commonly, $Z_{\text {eff }}$ measurement is performed by the use of LACs measured by $\mathrm{X}$-rays with two different energies. Thus, the use of synchrotron facilities where can generate monochromatic X-ray is one of the best methods for $Z_{\text {eff }}$ measurement [3]. However, synchrotrons are too large to install in general hospitals. Alternative methods are a photon-counting CT or a fast $\mathrm{kVp}$ switching dual-energy CT. Photon-counting CT uses X-ray detectors which can measure the energy of $\mathrm{X}$-ray. However, photon-counting CT has a limitation in counting rate which is less than $0.5 \times 10^{6} \mathrm{~s}^{-1}$ in general [4]. Typical number of X-rays coming into a detector in clinical practice is $10^{9} \mathrm{~mm}^{-2} \cdot \mathrm{s}^{-1}$ when they pass the air only and $10^{6}$ $\mathrm{mm}^{-2} \cdot \mathrm{s}^{-1}$ when they pass a thick body [5]. In this stage, photon-counting CT is not practical in view of counting rate problem. Fast $\mathrm{kVp}$ switching dual-energy $\mathrm{CT}$ requires two projection data on each measurement direction with switching high and low voltages within $10 \mathrm{~ms}$ [6]. Two transmission measurements are performed by X-rays with two different averaged energies. The difference between the two averaged energies is, however, not very large: with 80 and 140 $\mathrm{kVp}$, the typical averaged energies at the exit of X-ray tube are 41.4 and 58.9 $\mathrm{keV}$, respectively. According to the transmission direction of a human body, the averaged energy changes and results in beam-hardening artifact in CT im- 
age.

To overcome the problems associated with photon-counting CT and dualenergy CT, we have proposed a novel energy-resolved CT by using a transXend detector [7]. The schematic drawing of the transXend detector is shown in Figure 1 of [7]. The transXend detector consists of several segmented detectors aligned in the X-ray incident direction and measures X-rays as electric currents. The X-ray energy distribution is given after analysis using pre-estimated response function (RF). The transXend detector gives photon numbers in arbitrary energy ranges. Since X-rays are measured as electric currents by the transXend detector, there are no problems associated with the counting rate. This transXend detector collects transmission data of an object under study by repeating rotation and transverse movements. Thus, we called this detector the one-dimensional (1D) transXend detector. Yamashita et al. reported that $Z_{\text {eff }}$ of aluminum was measured within $1 \%$ error, where the error was defined as $\left(Z_{\text {eff }}-Z\right) / Z$ [8]. Kanno et al. also reported that relative error of measured $Z_{\mathrm{eff}} \mathrm{s}$ of water and acrylic were within 3\% [9]. Both studies were performed by the 1D transXend detector.

For the application of two-dimensional (2D) transXend detector for clinical practice in the future, the authors invented stripe absorbers which are placed in front of a flat panel detector (FPD) [10]. The stripe absorbers consist of two kinds of metal ribbons and provides four different X-ray energy spectra. Kanno et al. reported the possibility of 2D transXend detector using a thermo-luminescent plate [11].

As mentioned above, $Z_{\text {eff }}$ measurements for aluminum, acrylic, and water using the $1 \mathrm{D}$ transXend detector were conducted previously. In this study, we performed $Z_{\text {eff }}$ measurements for eleven human tissue-equivalent materials by the energy-resolved CT using the 2D transXend detector with a FPD. The eleven materials were grouped into four material categories: LUNG, SOFT TISSUE,

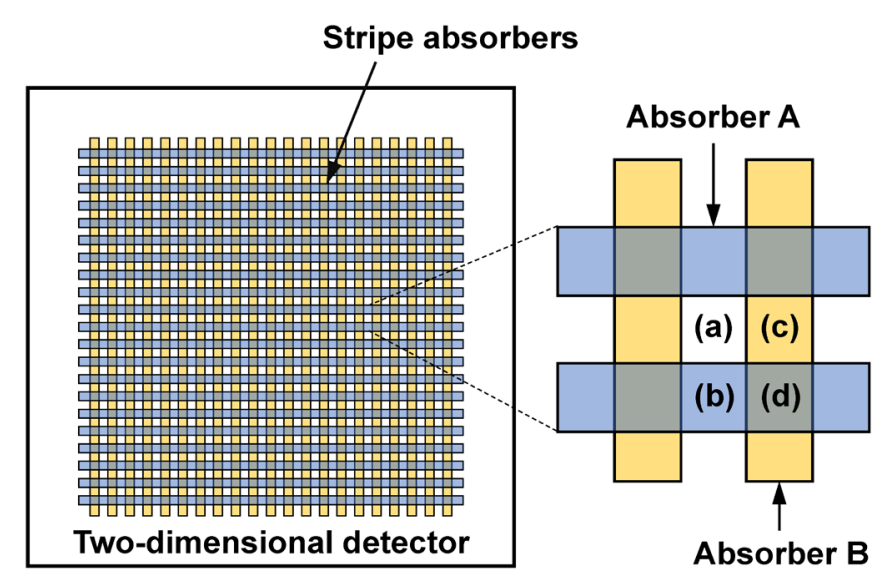

Figure 1. Schematic drawing of the two-dimensional transXend detector system with the stripe absorbers [11]. In region (a), X-rays directly enter to the FPD, without passing two absorbers. In region (b), (c), and (d), X-rays passed through the absorber A, B, and A + $\mathrm{B}$, respectively. 
SOFT BONE, and BONE. $Z_{\text {eff }}$ s were measured by using the RFs estimated under two different scenarios: With RFs estimated for the same materials with the one used for CT measurements, or with RFs estimated for different materials in each material category.

\section{Materials and Methods}

\subsection{Theory of an Effective Atomic Number Measurement}

Spiers et al. proposed the definition of $Z_{\text {eff }}$ for considering the X-ray absorption by human tissue [12]:

$$
Z_{\text {eff }}=\sqrt[2.94]{\sum_{k} \alpha_{k} Z_{k}^{2.94}}
$$

where $\alpha_{k}$ is the electron number fraction, and $Z_{k}$ is the atomic number of element $k$.

Torikoshi et al. showed the measurement of $Z_{\text {eff }}$ with two different monochromatic X-ray energies using a synchrotron facility [3]. In the energy range for $\mathrm{X}$-ray CT, 80 - $140 \mathrm{keV}$, the X-ray LAC of element $Z$ for monochromatic energy $E$ can be written as

$$
\mu(E)=\rho_{e}\left\{Z^{4} F(E, Z)+G(E, Z)\right\} .
$$

Here $\rho_{e}$ is the electron density, $\rho_{e} Z F(E, Z)$ is the photoelectric term, and $\rho_{e} G(E, Z)$ is the scattering term. Thus, atomic number $Z$ can be described by the ratio of LAC:

$$
f(Z)=\mu\left(E_{a}\right) / \mu\left(E_{b}\right) .
$$

Since the LAC of elements are summarized as a function of X-ray energy in the table of the National Institute of Standards and Technology (NIST), the term $f(Z)$ can be drawn by plotting the value of $\mu\left(E_{a}\right) / \mu\left(E_{b}\right)$ as a function of $Z$ [13]. Therefore, the $Z_{\text {eff }}$ can be obtained by using the Equation (3) with measuring LACs at two different X-ray energies $E_{a}$ and $E_{b}$. Since a CT image is the distribution of LAC, the ratio can be obtained by dividing two CT images which were acquired by two different monochromatic X-ray energies $E_{a}$ and $E_{b}$.

\subsection{The Relationship between the Measured Currents and X-Ray Energy Distribution}

When the transXend detector is used for X-ray transmission measurement, the relationship between the measured electric currents and the X-ray energy distribution is expressed in terms of following matrix equation [7]:

$$
\left(\begin{array}{c}
I_{1} \\
I_{2} \\
\vdots \\
I_{m}
\end{array}\right)=\left(\begin{array}{cccc}
R_{1,1} & R_{1,2} & \cdots & R_{1, n} \\
R_{2,1} & R_{2,2} & & \vdots \\
\vdots & & \ddots & \\
R_{m, 1} & \cdots & & R_{m, n}
\end{array}\right)\left(\begin{array}{c}
Y_{1} \\
Y_{2} \\
\vdots \\
Y_{n}
\end{array}\right) .
$$

Here $I_{i}(i=1, m)$ is the electric current value measured by $i$-th segmented detector, $Y_{j}(j=1, n)$ is the number of X-rays in the energy range $E_{j}$ and $R_{i, j}$ is the 
RF of the $i$-th segment detector in the energy range $E_{j}$ The X-ray energy distribution is obtained by solving Equation (4) using an unfolding code, such as SAND II [14]. In the unfolding process, the number of energy ranges and the widths of energy ranges can be assigned according to the materials of interest. More detailed information is described in elsewhere [15].

\subsection{Two-Dimensional transXend Detector}

For the clinical application of the transXend detector, a 2D transXend detector should be developed. To make the transXend detector system two-dimensional, we used a FPD and stripe absorbers which consist of two different absorbers A and $B$ in a lattice shape, as shown in Figure 1 [9]. With the stripe absorbers placed in front of the FPD, four different regions, (a)-(d), can be made, as shown in Figure 1. In region (a), X-rays enter to the FPD without passing two absorbers. Subsequently, in region (b), (c), and (d), X-rays passed through the absorber $\mathrm{A}, \mathrm{B}$, and $\mathrm{A}+\mathrm{B}$, respectively. We used $1 \mathrm{~mm}$-wide and 0.1-mm-thick tin and copper for absorber A and B, respectively. Calculated X-ray spectra arriving at FPD pixels in each region are shown in Figure 2. Considering the four regions as one pixel, each region has the role of segmented detectors. X-ray energy distribution can be acquired for the 2D position on the FPD by unfolding electric currents measured by the four regions. The employed FPD was Remote RadEye2 (Teledyne Rad-icon Imaging, Sunnydale, CA, USA) with pixel matrix of $1024 \times$ 1024 pixel $^{2}$ (active area $49.3 \times 49.2 \mathrm{~mm}^{2}$ ). The pixel size of photodiode was $48 \times$ $48 \mu \mathrm{m}^{2}$. Incident X-ray photons are absorbed by a $\mathrm{Gd}_{2} \mathrm{O}_{2} \mathrm{~S}$ scintillator plate and scintillation photons are detected by a 2D CMOS photodiode array.

\subsection{Human Tissue-Equivalent Materials}

Eleven RMI rods (Gammex, Middleton, WI, US) were used in this study, as summarized in Table 1. Diameter and height of each rod was $28 \mathrm{~mm}$ and 70 $\mathrm{mm}$, respectively. RMI rods were often used as the calibration materials of CT

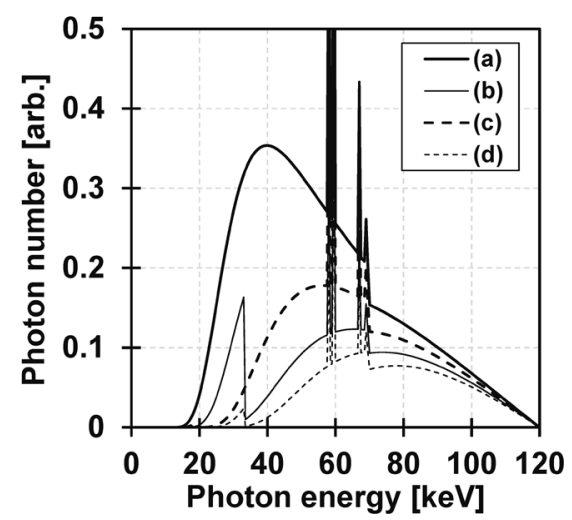

Figure 2. $120 \mathrm{kV}$-tube voltage $\mathrm{X}$-ray energy spectra passed through the (a) air, (b) tin (0.1-mm-thick), (c) copper (0.1-mm-thick), and (d) tin + copper. 
Table 1. Characteristics for RMI rods.

\begin{tabular}{ccccc}
\hline $\begin{array}{c}\text { Material } \\
\text { category }\end{array}$ & Rod material & $\begin{array}{c}\text { Relative electron } \\
\text { density }\end{array}$ & $\begin{array}{c}\text { Mass density } \\
{\left[\mathrm{g} / \mathrm{cm}^{3}\right]}\end{array}$ & $\begin{array}{c}\text { Theoretical } \\
\text { effective atomic } \\
\text { number }\end{array}$ \\
\hline \multirow{2}{*}{ LUNG } & LN300 & 0.29 & 0.29 & 7.86 \\
& LN450 & 0.45 & 0.46 & 7.84 \\
& Adipose & 0.92 & 0.94 & 6.40 \\
SOFT TISSUE & Breast & 0.96 & 0.98 & 7.24 \\
& Solid water & 0.99 & 1.02 & 8.11 \\
& Brain & 1.05 & 1.05 & 6.31 \\
SOFT BONE & Inner bone & 1.10 & 1.14 & 10.9 \\
& Bone mineral & 1.11 & 1.15 & 10.9 \\
& CB2-30\% & 1.28 & 1.33 & 11.4 \\
BONE & CB2-50\% & 1.47 & 1.56 & 13.0 \\
& Cortical bone & 1.70 & 1.82 & 14.1 \\
\hline
\end{tabular}

number-mass density conversion table for the dose distribution calculation in radiotherapy treatment planning. In such calibration, each rod was inserted to a 330-mm-diameter and 50-mm-height RMI phantom and all rods were scanned simultaneously. In this study, however, each rod was scanned individually to measure its $Z_{\text {eff }}$ for avoiding the fan-beam effect.

\subsection{Experiment}

Experimental set up was shown in Figure 3. As described in the previous paper, $\mathrm{RF}$ measurement for the phantom material was necessary prior to CT measurement [7]. Used materials for RF measurement were the same ones with the phantom for CT measurement: slabs of different thicknesses were prepared for each material in the phantom. We, however, transmission data for the RF estimation by a numerical calculation using Lambert-Beer's law since each rod was uniform:

$$
I=I_{0} \exp (-\mu t) .
$$

Here $I$ is photon number transmitted through a material with LAC, $\mu$, with thickness $t, I_{0}$ is incident photon number. Transmission data was calculated for each rod material with thicknesses ranging from 0 to $30 \mathrm{~mm}$ at intervals of 5 $\mathrm{mm} . I_{0}$ was normalized to the measured current induced by X-rays which passed the air only. In transXend analysis, RF data was interpolated by $1 \mathrm{~mm}$ interval. Scatter X-rays were not considered in the calculation.

After calculating transmission data for the RF estimation, each rod was scanned from one direction by the X-rays. Employed X-ray tube was ERESCO MF4 (GE Sensing \& Inspection Technologies, Ahrensburg, Germany) with a tungsten target and built-in filters made from 0.8 -mm-thick beryllium and 2-mm-thick aluminum. The X-ray tube was placed $1000 \mathrm{~mm}$ away from the FPD. The X-ray tube operating conditions were $120 \mathrm{kV}$ for tube voltage, $2.3 \mathrm{~mA}$ 


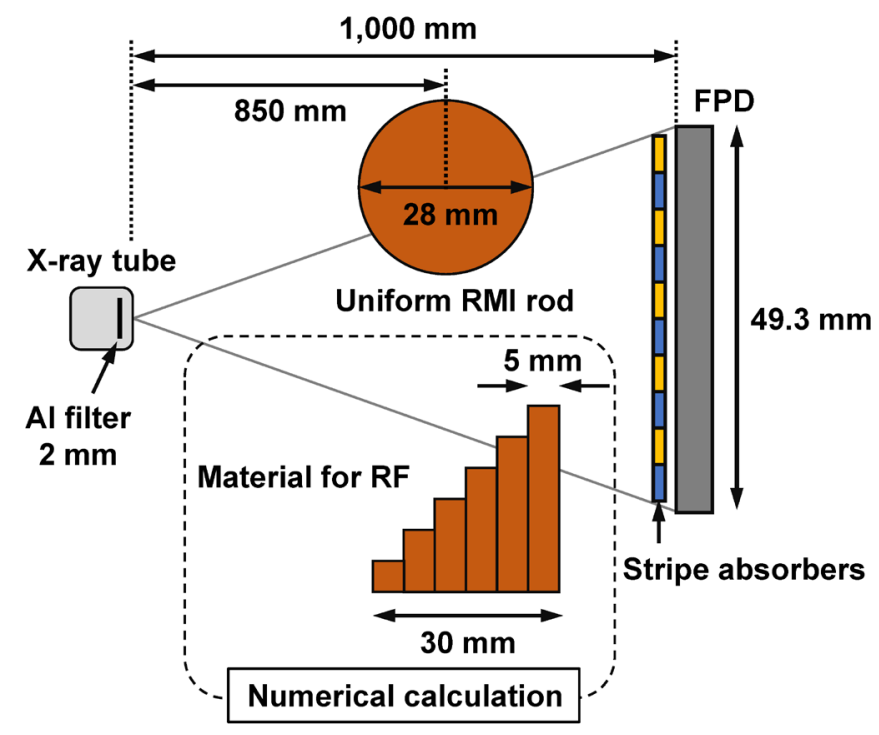

Figure 3. Experimental setup for two-dimensional transXend detector system with the stripe absorbers. Response functions (RFs) of each rod material were calculated numerically.

for tube current, and $1 \mathrm{~s}$ for exposure time. Since each rod was axial symmetry, projection data for each rod was duplicated 359 times to make $1^{\circ}$ step data. Source-to-axis of the phantom distance was $850 \mathrm{~mm}$.

Six energy ranges were defined for obtaining X-ray energy distribution, as shown in Table 2. Since almost no X-rays with the energy under $15.0 \mathrm{keV}$ entered into the FPD, those X-rays were excluded from the analysis. The X-rays in the energy range $E_{2}: 35.0-36.0 \mathrm{keV}$ and $E_{5}: 65.0-66.0 \mathrm{keV}$ were used as pseudo-monochromatic X-rays. Measured currents in the center column of the FPD were unfolded by SAND II code to obtain energy distributions. Number of $\mathrm{X}$-rays in each energy ranges were estimated for each projection. With $Y_{2}$ and $Y_{5}$, CT images were reconstructed by maximum likelihood-expectation maximization method [16]. Iterative number was 30 times which was optimized prior to the measurements. With the LAC data table of NIST, $Z-\mu\left(E_{2}\right) / \mu\left(E_{5}\right)$ relationship can be drawn as Figure 4. CT images of $\mu\left(E_{2}\right)$ and $\mu\left(E_{5}\right)$ for each rod were converted to a $Z_{\text {eff }}$ image using Figure 4.

In previous studies [7] [8], the RF was estimated by using the same material with the one of phantom for CT measurement. In this study, we demonstrated two different analysis scenarios. In the first scenario, we employed the RF estimated for the same material as the one of the phantom for CT measurement (self-RF scenario). In the second scenario, we employed the RF estimated for the different material than the one of the phantom for CT measurement (cross-RF scenario). The purpose of the cross-RF scenario was to determine the representative rod materials in each material category. Reduction of the number of materials for RF estimation would widen the application of energy-resolved CT using 2D transXend detector. Mean and standard deviation (SD) of $Z_{\text {eff }}$ for each rod material was calculated in $10 \times 10 \mathrm{~mm}^{2}$ region-of-interest on $Z_{\text {eff }}$ image. 


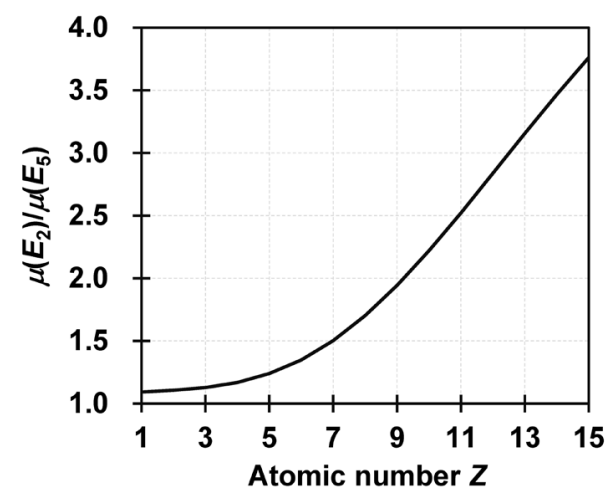

Figure 4. The $Z-\mu\left(E_{2}\right) / \mu\left(E_{5}\right)$ relationship between atomic number, $Z$, and the linear attenuation coefficient ratio, $\mu\left(E_{2}\right) / \mu\left(E_{5}\right)$, obtained by two different $\mathrm{X}$-ray energy ranges; $E_{2}: 35.0-36.0 \mathrm{keV}$ and $E_{5}: 65.0-66.0 \mathrm{keV}$.

Table 2. Assigned energy ranges. Unit: [keV].

\begin{tabular}{cccccc}
\hline$E_{1}$ & $E_{2}$ & $E_{3}$ & $E_{4}$ & $E_{5}$ & $E_{6}$ \\
\hline $15.0-35.0$ & $35.0-36.0$ & $36.0-60.0$ & $60.0-65.0$ & $65.0-66.0$ & $66.0-120.0$ \\
\hline
\end{tabular}

\section{Results}

\subsection{Self-Response Function Scenario}

Measured $Z_{\text {eff }} \mathrm{s}$ for material categories of LUNG and SOFT TISSUE, and SOFT BONE and BONE were plotted in Figure 5(a) and Figure 5(b), respectively, by white circle with error bars. Error bars for SOFT BONE and BONE were smaller than those of other material categories. As shown in Figure 5(a) and Figure 5(b), mean of measured $Z_{\text {eff }}$ for each rod material were underestimated systematically. Relative errors of measured $Z_{\text {eff }}$ to theoretical ones were shown in Figure 5 (c) by white squares. Median relative error of measured $Z_{\text {eff }}$ was $-6.92 \%$ (range: $-7.89 \%--4.60 \%$ ). Thus, we normalized each measured $Z_{\text {eff }}$ to the one of Breast, 7.24, with multiplying the value of 1.07, as shown in Figure 5(a) and Figure 5(b) by orange circles. $Z_{\text {eff }}$ s for all rod materials had close values to theoretical ones, and median relative error of normalized $Z_{\text {eff }}$ was $-0.75 \%$ (range: $-1.79-+1.73 \%$ ). Relative errors of normalized $Z_{\text {eff }} \mathrm{s}$ were shown in Figure 5(c) by orange squares.

\subsection{Cross-Response Function Scenario}

We also estimated $Z_{\text {eff }}$ for each rod material using the RF obtained for different rod materials in the same material category. Normalized $Z_{\text {eff }}$ for material categories of LUNG and SOFT TISSUE, and of SOFT BONE and BONE in the cross-RF scenario were shown in Figure 6(a), and Figure 6(b), respectively. Square plots represented the normalized $Z_{\text {eff }}$ for each rod in the cross-RF scenario.

In the material category of LUNG, relative error of $Z_{\text {eff }}$ of LN450 with the RF 


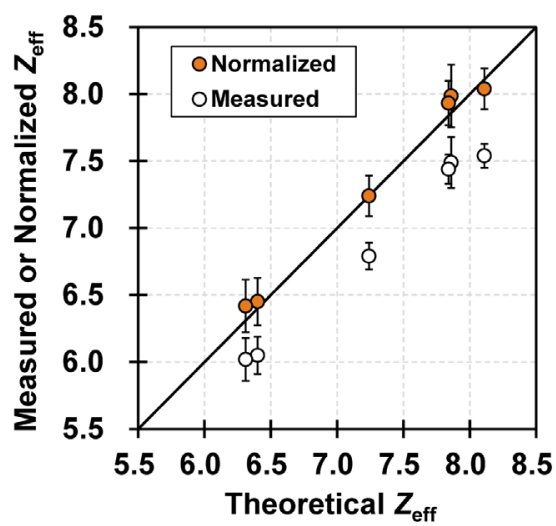

(a)

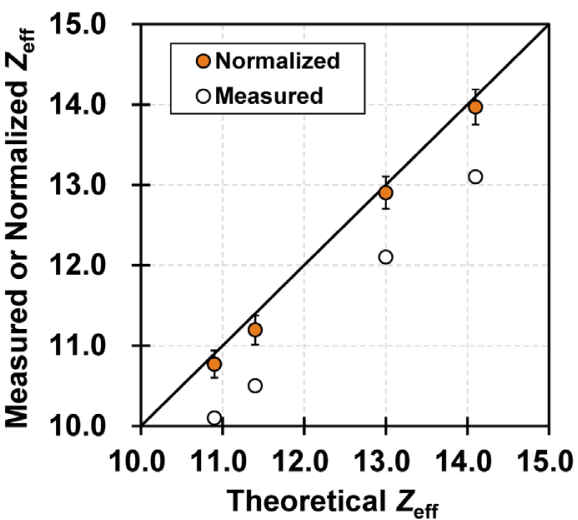

(b)

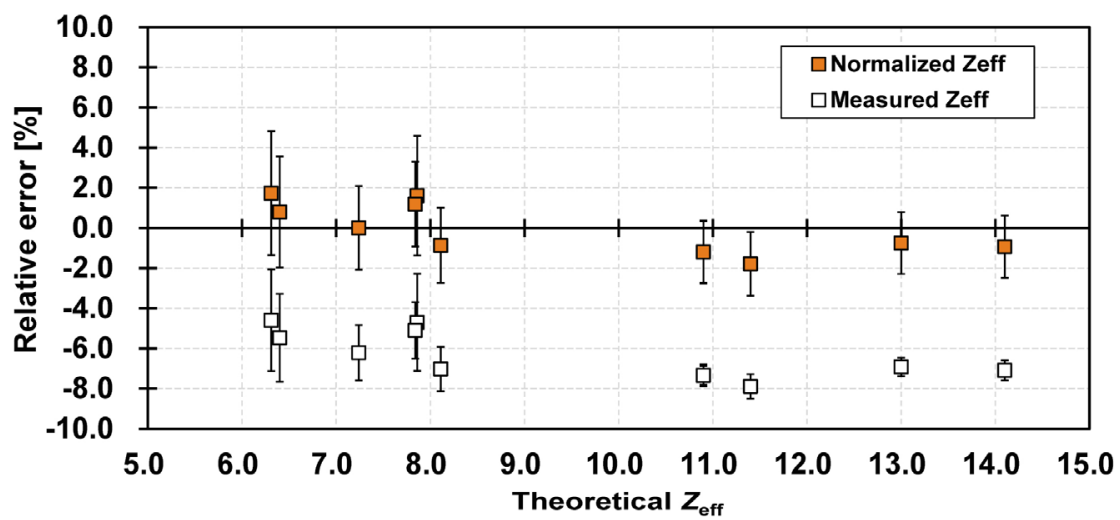

(c)

Figure 5. Theoretical effective atomic number $\left(Z_{\text {eff }}\right)$ versus measured and normalized one for material categories of (a) LUNG and SOFT TISSUE, and for (b) SOFT BONE and BONE in the self-response function (RF) scenario. (c) Relative error of measured and normalized $Z_{\text {eff }}$ to theoretical one.

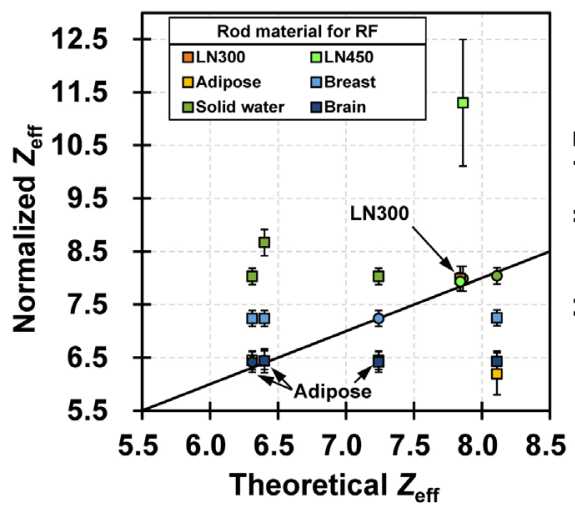

(a)

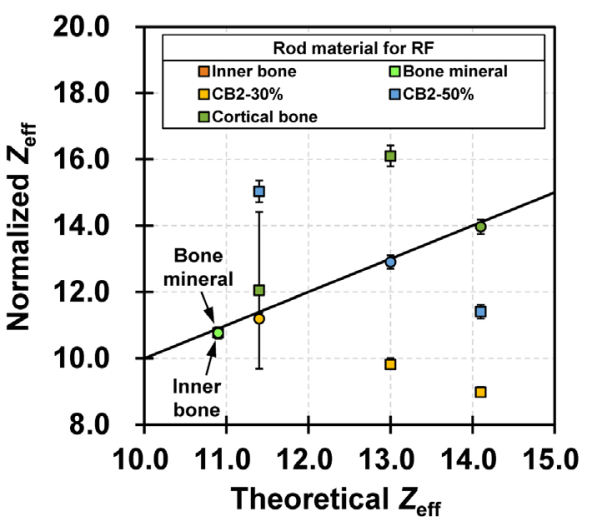

(b)

Figure 6. Theoretical effective atomic number $\left(Z_{\text {eff }}\right)$ versus normalized one for material categories of (a) LUNG and SOFT TISSUE, and for (b) SOFT BONE and BONE in the cross-response function (RF) scenario. Each circle plot represent the normalized $Z_{\text {eff }}$ obtained by the self-RF scenario.

of LN300, or $Z_{\text {eff }}(\mathrm{LN} 450<\mathrm{LN} 300)$, to theoretical one was $1.87 \%$, while that of $Z_{\text {eff }}$ $(\mathrm{LN} 300<\mathrm{LN} 450)$ was $43.8 \%$. In the material category of SOFT BONE, relative 
errors of $Z_{\text {eff }}$ (Bone mineral $\leftarrow$ Inner bone) and $Z_{\text {eff }}$ (Inner bone $\leftarrow$ Bone mineral) were both $-1.20 \%$. In the material category of SOFT TISSUE, relative errors of $Z_{\text {eff }}\left(\right.$ Brain $\leftarrow$ Adipose) and $Z_{\text {eff }}$ (Adipose $\leftarrow$ Brain) were $2.23 \%$ and $0.63 \%$, respectively, while relative errors of $Z_{\text {eff }}$ of other rod materials for the cross-RF scenario in the SOFT TISSUE category were over $10 \%$. In the material category of BONE, although relative errors of $Z_{\text {eff }}(\mathrm{CB} 2-30 \% \leftarrow$ Cortical bone) was $5.69 \%$, error bar of $Z_{\text {eff }}(\mathrm{CB} 2-30 \% \leftarrow$ Cortical bone) was large, as shown in Figure 6(b). Relative errors of $Z_{\text {eff }}$ of other rod materials for the cross-RF scenario in the BONE category were over $19 \%$.

\section{Discussion}

This was the first study to conduct $Z_{\text {eff }}$ measurement by the $2 \mathrm{D}$ transXend detector with the FPD. Upon the self-RF scenario, error bars for SOFT BONE and BONE were smaller than those of other material categories. It is because that the slope of $Z-\mu\left(E_{2}\right) / \mu\left(E_{5}\right)$ relationship around $Z=13$ is steeper than that around $Z=$ 7, as shown in Figure 4. Thus, uncertainty of atomic numbers for SOFT BONE and BONE material categories to the $\mu\left(E_{2}\right) / \mu\left(E_{5}\right)$ are smaller than those for other material categories. For instance, in $Z-\mu\left(E_{2}\right) / \mu\left(E_{5}\right)$ relationship in Figure 4, if the atomic number is varied from $Z=7$ to $Z=8$, the $\mu\left(E_{2}\right) / \mu\left(E_{5}\right)$ changes by 0.2 . If the $\mu\left(E_{2}\right) / \mu\left(E_{5}\right)$ of $Z=13$ increases by 0.2 , the result would be $Z=13.6$.

In this study, $Z_{\text {eff }}$ s for each rod material were measured within $\pm 1.8 \%$ error after the normalization using the $2 \mathrm{D}$ transXend detector with numerically-estimated RF data. Until now, the dedicated experiments before the CT measurement had been necessary for the estimation of the RF data and had been a time-consuming process. Exclusion of dedicated RF experiments will contribute to the clinical application of the 2D transXend detector. Moreover, the result of this study is comparable to the previous studies which measured $Z_{\text {eff }} \mathrm{s}$ for aluminum, acrylic, and water using 1D transXend detector with experimentally-estimated RF data within 1\% - 3\% error [7] [8].

Bazalova et al. demonstrated $Z_{\text {eff }}$ measurement for RMI rods with a clinical CT scanner with two different X-ray tube voltages [17]. The cited authors reported that mean $\pm \mathrm{SD}$ of relative error of measured $Z_{\mathrm{eff}} \mathrm{s}$ was $2.8 \% \pm 2.6 \%$, and the largest error was $12.0 \%$. Goodsitt et al. conducted $Z_{\text {eff }}$ measurement for RMI rods with a rapid $\mathrm{kVp}$ switching dual energy CT scanner [18]. In their study, median relative error of measured $Z_{\text {eff }} s$ for the same rod materials with our study was $-3.49 \%$ (range: $-4.93 \%-+15.1 \%$ ), while that of our study was $-0.75 \%$ (range: $-1.79 \%-+1.73 \%$ ). Note that $Z_{\text {eff }}$ for LUNG category were excluded from the analysis in the study of Goodsitt et al. because those were unmeasurable by the CT scanner they used [18]. Although the cited authors scanned all rods at the same time, while we scanned each rod individually, normalized $Z_{\text {eff }} \mathrm{s}$ obtained by the energy-resolved CT with 2D transXend detector showed good agreement with the theoretical $Z_{\text {eff }}$ s.

AAPM Task Group No. 65 recommended that the uncertainty of patient data 
should be within $1.5 \%$ for MV photon dose calculation [19]. In our study, $Z_{\text {eff }}$ for all rod materials, except for LN300, Brain, and CB2-30\%, were measured within $1.5 \%$ error that satisfied the recommendation. To improve the $Z_{\text {eff }}$ measurement accuracy, scattered X-ray should be considered in the RF estimation by using Monte Carlo simulation [20].

Upon the cross-RF scenario, relative error of $Z_{\text {eff }}(\mathrm{LN} 300<\mathrm{LN} 450)$ to theoretical one was larger than that of $Z_{\text {eff }}(\mathrm{LN} 450<\mathrm{LN} 300)$ in the material category of LUNG. This was because the large noise on the projection data of LN300 resulted from its low mass density. Thus, only LN300 would be the representative rod material for the material category of LUNG. In the material category of SOFT BONE, both Inner bone and Bone mineral rods would be the representative ones. However, in the material category of SOFT TISSUE and BONE, no rod materials which can represent all other rod materials in each material category were found. Since the relative errors for $Z_{\text {eff }}$ (Brain $\leftarrow$ Adipose) and $Z_{\text {eff }}$ (Adipose $<$ Brain) were within $2.2 \%$, the RF of the representative rod material can be effective if the difference of $Z_{\text {eff }}$ between the representative rod material and measuring object is within 0.09 . This was because the RF depended on materials of measuring object. To overcome the problem, the RF which is independent from materials of measuring object should be employed. Recently, Maruyama et al. proposed a RF estimation method independent from materials of measuring object. They estimated the RF for the 1D transXend detector consisted of silicon only with using Monte Carlo simulation [21]. However, the RF estimation method by Maruyama et al. cannot be applied to a transXend detector with detector materials which K-edges are in the diagnostic X-ray energy. Since our FPD had $\mathrm{Gd}_{2} \mathrm{O}_{2} \mathrm{~S}$ scintillator with K-edge of gadolinium at $50.2 \mathrm{keV}$, we could not use the method proposed by Maruyama et al. Estimation of the RF for the transXend detector with K-edge material should be developed in the future study.

One of the limitations of this study was the effect of the fan or cone-beam geometry. With the 1D transXend detector, X-ray paths for each pixel on one projection data were the same. In the $2 \mathrm{D}$ transXend detector, however, X-ray paths for each pixel on one projection data were different due to fan or cone angle. In this study, we did not take into account of the effect.

\section{Conclusion}

$Z_{\text {eff }}$ measurements were performed by energy-resolved CT using the $2 \mathrm{D}$ transXend detector with numerically-estimated RF data. Eleven human tissue-equivalent materials were employed for $Z_{\text {eff }}$ measurements. Upon the self-RF scenario, the normalization was performed due to the systematic underestimation of measured $Z_{\text {eff }}$ s. Normalized $Z_{\text {eff }}$ for all rod materials were in good agreement with the theoretical ones. The numerically-estimated RF was shown useful for $Z_{\text {eff }}$ measurement: time-consuming experiment for RF estimation is not necessary. Upon the cross-RF scenario, LN300, and Inner bone and Bone mineral rod materials would be the representative rod materials for the material categories of 
LUNG, and SOFT BONE, respectively, while no representative rods were found in other material categories. To improve the accuracy of $Z_{\text {eff }}$ measurement, the $\mathrm{RF}$ estimation method which is independent from the measuring object and also can be applied to a transXend detector with K-edge material should be developed in the future study.

\section{Acknowledgements}

This research was supported, in part, by the Japan Society for the Promotion of Science (JSPS) Grant-in-Aid for JSPS Fellows (No. 16J08928).

\section{References}

[1] Chetty, I.J., Curran, B., Cygler, J.E., De Marco, J.J., Ezzell, G., Faddegon, B.A., et al. (2007) Report of the AAPM Task Group No. 105: Issues Associated with Clinical Implementation of Monte Carlo-Based Photon and Electron External Beam Treatment Planning. Medical Physics, 34, 4818-4853. https://doi.org/10.1118/1.2795842

[2] Verhaegen, F. and Devic, S. (2005) Sensitivity Study for CT Image Use in Monte Carlo Treatment Planning. Physics in Medicine \& Biology, 50, 937-946. https://doi.org/10.1088/0031-9155/50/5/016

[3] Toriloshi, M., Tsunoo, T., Sasaki, M., Endo, M., Noda, Y., Ohno, Y., et al. (2003) Electron Density Measurement with Dual-Energy X-ray CT Using Synchrotron Radiation. Physics in Medicine \& Biology, 48, 673-685. https://doi.org/10.1088/0031-9155/48/5/308

[4] Taguchi, K., Frey, E., Wang, X., Iwanczyk, J. and Barber, W. (2010) An Analytical Model of the Effects of Pulse Pileup on the Energy Spectrum Recorded by Energy Resolved Photon Counting X-Ray Detectors. Medical Physics, 37, 3957-3969. https://doi.org/10.1118/1.3429056

[5] Yu, L., Leng, S. and McCollough, C.H. (2012) Dual-Energy CT-Based Monochromatic Imaging. American Journal of Roentgenology, 199, S9-S15. https://doi.org/10.2214/AJR.12.9121

[6] Xu, C., Danielsson, M., Karlsson, S., Svensson, C. and Bornefalk, H. (2012) Preliminary Evaluation of a Silicon Strip Detector for Photon-Counting Spectral CT. Nuclear Instruments and Methods in Physics Research Section A, 677, 45-51. https://doi.org/10.1016/j.nima.2012.02.034

[7] Kanno, I., Imamura, R., Mikami, K., Uesaka, A., Hashimoto, M., Phtaka, M., et al. (2008) A Current Mode Detector for Unfolding X-Ray Energy Distribution. Journal of Nuclear Science and Technology, 45, 1165-1170. https://doi.org/10.1080/18811248.2008.9711905

[8] Yamashita, Y., Kimura, M., Hamaguchi, T., Kanno, I., Ohtaka, M., Hashimoto, M., et al. (2014) Measurement of Effective Atomic Numbers using Energy-Resolved Computed Tomography. Journal of Nuclear Science and Technology, 51, 12561263. https://doi.org/10.1080/00223131.2014.919881

[9] Kanno, I., Yamashita, Y., Kimura, M. and Inoue, F. (2015) Effective Atomic Number Measurement with Energy-Resolved Computed Tomography. Nuclear Instruments and Methods in Physics Research Section A, 787, 121-124. https://doi.org/10.1016/j.nima.2014.11.072

[10] Kanno, I., Yamauchi, K. and Hamaguchi, T. (2016) Two-Dimensional "transXend" Detector with Band Structure Absorbers for Third-Generation Energy-Resolved Computed Tomography with Improved Spatial Resolution. Journal of Nuclear 
Science and Technology, 54, 22-29. https://doi.org/10.1080/00223131.2016.1202154

[11] Kanno, I., Yamashita, Y., Kanai, E., Ogawa, T. and Shinsho, K. (2016) Two-Dimensional "TransXend" Detector for Third Generation Energy-Resolved Computed Tomography. Journal of Nuclear Science and Technology, 53, 258-262. https://doi.org/10.1080/00223131.2015.1037810

[12] Spiers, F.W. (1946) Effective Atomic Number and Energy in Tissues. The British Journal of Radiology, 19, 52-63. https://doi.org/10.1259/0007-1285-19-218-52

[13] Hubbell, J.H. and Seltzer, S.M. (2010) Tables of X-Ray Mass Attenuation Coefficients and Mass Energy-Absorption Coefficients from $1 \mathrm{keV}$ to $20 \mathrm{MeV}$ for Elements $Z=1$ to 92 and 48 Additional Substances of Dosimetric Interest. The Physical Measurement Laboratory, The National Institute of Standards and Technology, Gaithersburg, MD. https://www.nist.gov/pml/x-ray-mass-attenuation-coefficients

[14] McElroy, W., Berg, S., Crockes, T. and Hawkins, G.A. (1967) Computer-Automated Iterative Method for Neutron Fux Spectra Determination by Foil Activation. AFWL-TR-67-41. Air Force Weapons Laboratory, Albuqueque, NM.

[15] Imamura, R., Mikami, K., Minami, Y., Kanno, I., Ohtaka, M., Hashimoto, M., et al. (2010) Unfolding Method with X-Ray Path Length-Dependent Response Functions for Computed Tomography Using X-Ray Energy Information. Journal of Nuclear Science and Technology, 47, 1075-1082. https://doi.org/10.1080/18811248.2010.9711672

[16] Shepp, L.A. and Vardi, Y. (1982) Maximum Likelihood Reconstruction for Emission Tomography. IEEE Transactions on Medical Imaging, 1, 113-122. https://doi.org/10.1109/TMI.1982.4307558

[17] Bazalova, M., Carrier, J.F., Beaulieu, L. and Varhaegen, F. (2008) Dual-Energy CT-Based Material Extraction for Tissue Segmentation in Monte Carlo Dose Calculations. Physics in Medicine \& Biology, 53, 2439-2456. https://doi.org/10.1088/0031-9155/53/9/015

[18] Goodsitt, M.M., Christodoulou, E.G. and Larson, S.C. (2011) Accuracies of the Synthesized Monochromatic CT Numbers and Effective Atomic Numbers Obtained with a Rapid kVp Switching Dual Energy CT Scanner. Medical Physics, 38, 2222-2232. https://doi.org/10.1118/1.3567509

[19] Papanikolaou, N., Battista, J.J., Kappas, C., Klein, E., Mackie, T.R., Sharpe, M., et al. (2004) Tissue Inhomogeneity Corrections for Megavoltage Photon Beams. AAPM Report No. 65, 1-142.

[20] Tsai, T.S. and Kanno, I. (2016) A Simulation Study on the Influence of Scattered X-Rays in Energy-Resolved Computed Tomography. Journal of Nuclear Science and Technology, 54, 205-212. https://doi.org/10.1080/00223131.2016.1236709

[21] Maruyama, Y., Hamaguchi, T., Tsai, T.S. and Kanno, I. (2017) Response Function Estimation with Fine Energy Bins for the Energy-Resolved Computed Tomography Using a TransXend Detector. Journal of Nuclear Science and Technology, 55, 199208. https://doi.org/10.1080/00223131.2017.1389311 